\title{
Field-controlled phase-rectified magnonic multiplexer
}

\author{
C. S. Davies, ${ }^{1}$ A. V. Sadovnikov, ${ }^{2}$ S. V. Grishin, ${ }^{2}$ Yu. P. Sharaevsky, ${ }^{2}$ S. A. Nikitov,, 3 and V. V. Kruglyak ${ }^{1}$ \\ ${ }^{1}$ School of Physics, University of Exeter, Exeter, United Kingdom \\ ${ }^{2}$ Laboratory "Metamaterials", Saratov State University, Saratov, Russia \\ ${ }^{3}$ Kotel'nikov Institute of Radioengineering and Electronics, Russian Academy of Science, Moscow, Russia
}

\begin{abstract}
The mechanism used to alter the features of propagating spin waves is a key component underpinning the functionality of high frequency magnonic devices. Here, using experiment and micromagnetic simulations, we demonstrate the feasibility of a magnonic multiplexer in which the spin-wave beam is toggled between device output branches by the polarity of a small global bias magnetic field. Due to the anisotropy inherent to the dispersion of magnetostatic spin waves, the phase fronts of the output spin waves are asymmetrically tilted relative to the direction of the beam propagation (group velocity). We show how the phase tilts could be (partly) rectified in magnonic waveguides of variable width.
\end{abstract}

Index Terms — spin-wave caustics, field control, magnonics, multiplexer

\section{INTRODUCTION}

$\mathbf{I}_{\mathrm{e} e}^{\mathrm{T}}$ IS widely recognized that conventional CMOS-based electronics is beginning to plateau in its development [1], due to fundamental trade-offs associated with the processing speed, miniaturization etc. Attention is therefore turning towards magnetics-based technologies, including spintronics [2], magnonics [3], and magnon-spintronics [4], which might either offer alternative means of data processing and communication or complement semiconductor-based devices. In magnonics, spin waves are used as signal carriers within networks of magnonic waveguides, while a wide range of magnonic gates and devices [5]-[8] are being developed with the eventual aim of constructing all-spin-wave circuitry for signal and data processing [3].

The ability to control the propagation of spin waves in magnonic networks is crucial for further development of magnonic technology. The majority of control mechanisms used in magnonic waveguides to date involves the application of an external bias magnetic field. The strength of the internal effective magnetic field and the orientation of the magnetization both vary as a function of the applied magnetic field and in turn dictate the spin wave frequency, wave vector, and more generally, the spin wave dispersion in the waveguide [9]. The bias magnetic field can be applied either locally or globally. Local magnetic fields are most easily generated by current-carrying elements, and are frequently used e.g. to excite spin waves [10]-[11]. However, current carrying elements do not lend themselves well to miniaturization, as the inevitable Ohmic losses and impedance matching become increasingly difficult to control upon down-scaling: indeed, this is already a major barrier to the further development of conventional electronics.

It might be preferable to use less strongly localized (global on the length scales of individual device components [12]) magnetic fields, as this option does not necessitate a large complex network of carefully positioned wires and cooling

Manuscript received March 18, 2015. Corresponding author: C. S. Davies (e-mail: csd203@exeter.ac.uk).

Digital Object Identifier inserted by IEEE elements. The use of global and therefore virtually uniform magnetic fields means that the local control of spin-wave propagation is instead to be achieved through spatial inhomogeneities of alternative nature [5]. The two options available in the context of all-spin-wave circuitry are the geometry and material composition of the magnonic waveguides.

The specific device type studied in this paper is the spin wave multiplexer. In the $\mathrm{Y}$-shaped Permalloy magnonic multiplexer designed by Vogt et al [8], the spin-wave propagation path was controlled by a constant electrical current in a conducting underlayer. This current could be switched to flow underneath and therefore to deliver a transverse bias magnetic field across either branch of the multiplexer. The spin waves in the multiplexer were then guided along the magnetized branch. However, a significant limitation of this device lies in its reliance on local currentcarrying wires, for the reasons already discussed.

In this Letter, we use Brillouin Light Scattering (BLS) imaging and micromagnetic calculations to demonstrate the functionality of a magnonic multiplexer the output branch of which is toggled by the polarity of a small global bias magnetic field. The output spin-wave phase fronts from the multiplexer tend to be tilted due to the anisotropy of the magnetostatic spin-wave dispersion. Using simulations, we show how the phase velocity of the spin waves can be (at least partly) realigned with their group velocity.

\section{Methodology}

The sample consisted of three magnonic waveguides joined to form a Y-shaped multiplexer, as shown in Fig. 1 (a). The multiplexer was fabricated by laser-scribing a $7.5 \mu$ m-thick film of yttrium-iron garnet (YIG) grown on a substrate of gadolinium-gallium garnet. The saturation magnetization of YIG was $140 \mathrm{G}$. The lateral widths of the waveguides were $470 \mu \mathrm{m}$, and the "leg" and "arms" of the multiplexer were inclined at $42^{\circ}$ relative to each other. A uniform bias field $\boldsymbol{H}_{\mathrm{B}}$, of strength $1160 \mathrm{Oe}$, was applied in the plane of the sample at an angle of $7^{\circ}$ to the leg of the multiplexer. 
To excite spin waves, a microstrip transducer was positioned on the upper surface of the YIG film at the base of the multiplexer leg, approximately $2.4 \mathrm{~mm}$ away from the legarm junction. The microstrip was loaded with a microwave electrical current, and the BLS technique (described in detail e.g. in Refs. [13]-[14]) was used to image the propagation path of spin waves excited at the microwave frequency at different time delays after the onset of the microwave.
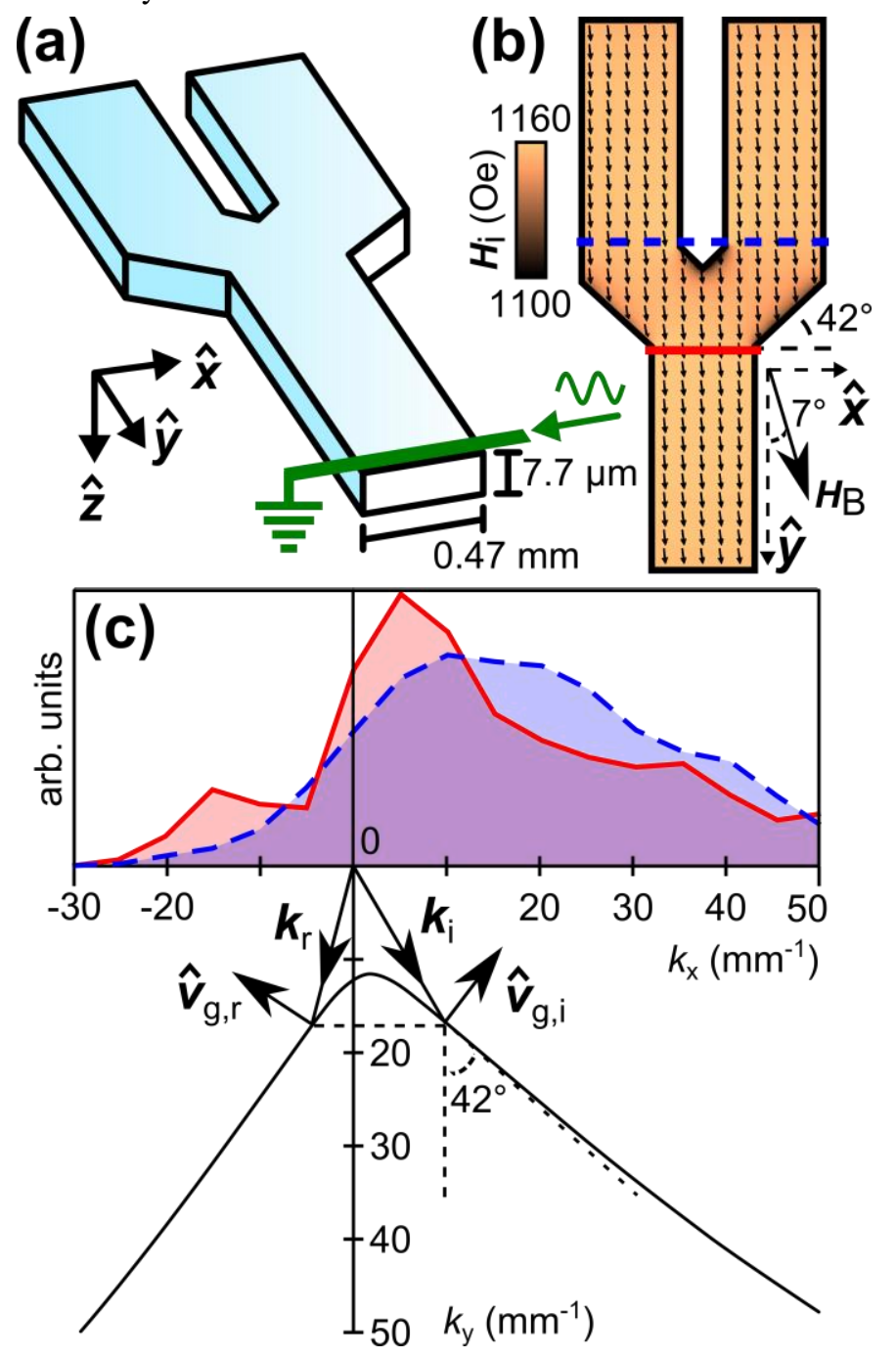

Fig. 1 (color online) (a) The studied sample is schematically shown. (b) The calculated projection of the internal magnetic field onto the magnetization (color scale) and local orientation of the magnetization (arrows) are shown for the ground magnetic state of the multiplexer. (c) Upper panel: The spinwave spectral power distribution calculated from dynamic micromagnetic simulations is shown for the top section of the leg (solid red) and the base of the straight section of the arm (dashed blue), as indicated in (b). Lower panel: The isofrequency curve for dipolar spin waves of the lowest thickness order is shown for the frequency of $5.09 \mathrm{GHz}$. Typical unit vectors for the incident and reflected spin-wave wave vectors ( $\boldsymbol{k}_{\mathrm{i}}$ and $\boldsymbol{k}_{\mathrm{r}}$ respectively) and group velocities $\left(\boldsymbol{v}_{\mathrm{g}, \mathrm{i}}\right.$ and $\boldsymbol{v}_{\mathrm{g}, \mathrm{r}}$ respectively) are also shown.

The experiments were modelled using micromagnetic simulations performed with the Object-Oriented MicroMagnetic Framework (OOMMF) [15]. The simulated sample had a geometry designed to mimic the sample studied experimentally. We assumed the gyromagnetic ratio of $2.80 \mathrm{MHz} / \mathrm{Oe}$, the saturation magnetization of $140 \mathrm{G}$, and the Gilbert damping coefficient of $1 \times 10^{-4}$. The multiplexer was enclosed within a cuboid of size $1.24 \mathrm{~mm} \times 3.34 \mathrm{~mm}$ $\times 7.5 \mu \mathrm{m}$, and discretized into a mesh of $10 \mu \mathrm{m} \times 10 \mu \mathrm{m} \times$ $2.5 \mu \mathrm{m}$ elements. Due to the macroscopic dimensions of the sample and the experimental resolution of BLS being far in excess of the exchange length (approximately $10 \mathrm{~nm}$ ), the exchange interaction was neglected in the simulations.

The micromagnetic simulations were performed in two stages [16]. First, static simulations were run to obtain the ground magnetic state of the multiplexer at $\boldsymbol{H}_{\mathrm{B}}=1160 \mathrm{Oe}$. The calculated configuration, in terms of the distributions of the projection of the internal field $\left(\boldsymbol{H}_{\mathrm{i}}\right)$ onto the static magnetization and of the magnetization orientation (averaged over $10 \times 10$ mesh cells), is shown in Fig. 1 (b). The effective magnetic field and magnetization configurations were found to be highly uniform across the multiplexer, apart perhaps from its edge regions. So, in the following analysis, we assumed the internal magnetic field to be equal to the applied magnetic field of 1160 Oe. In particular, we used the dipolar dispersion relation from Ref. [9] to calculate the isofrequency curve for the frequency $f=5.09 \mathrm{GHz}$ (lower panel of Fig. 1 (c)), where $\frac{\partial k_{x}}{\partial k_{y}} \approx 0.9$ for $40 \mathrm{~mm}^{-1} \geq k_{\mathrm{x}} \geq 5 \mathrm{~mm}^{-1}$.

In the second stage of the micromagnetic simulations, spin waves were excited at the base of the multiplexer leg by a local magnetic field of either pulsed $\left(h_{\mathrm{p}}\right)$ or harmonic $\left(h_{\mathrm{cw}}\right)$ temporal profile, which were defined respectively as

$$
h_{\mathrm{p}}=h_{\mathrm{p}, 0} \frac{\sin \left(2 \pi f_{\mathrm{c}}\left(t-t_{0}\right)\right)}{2 \pi f_{\mathrm{c}}\left(t-t_{0}\right)}\left(h_{k q, y} \hat{\mathrm{y}}+h_{k q, z} \hat{\mathrm{z}}\right)
$$

and

$$
h_{c w}=h_{c w, 0} \sin (2 \pi f \cdot t)\left(h_{k q, y} \hat{\boldsymbol{y}}+h_{k q, z} \hat{z}\right) \text {. }
$$

The spatial distribution of the fields was calculated in both cases from the Karlqvist equations [17] as

$$
\begin{aligned}
& h_{k q, y}(y, z)=\frac{1}{\pi}\left(\tan ^{-1}\left[\frac{w+2 y}{2 z}\right]+\tan ^{-1}\left[\frac{w-2 y}{2 z}\right]\right) \\
& h_{k q, z}(y, z)=\frac{1}{\pi} \ln \left[\frac{(w / 2-y)^{2}+z^{2}}{(w / 2+y)^{2}+z^{2}}\right] .
\end{aligned}
$$

The amplitudes of the dynamic pulsed and harmonic fields were $h_{\mathrm{p}, 0}=5 \mathrm{Oe}$ and $h_{\mathrm{cw}, 0}=0.1 \mathrm{Oe}$, respectively. The cut-off frequency of the sinc-function spectrum was $f \mathrm{c}=10 \mathrm{GHz} . f$ was the frequency of the microwave excitation, and the width of the microstrip antenna $w$ was $30 \mu \mathrm{m}$.

\section{RESULTS}

Electrically measured spin-wave transmission characteristics of straight YIG stripes of $470 \mu \mathrm{m}$ width and 
$7.5 \mu \mathrm{m}$ thickness magnetized by a field of 1160 Oe revealed that the propagation of spin waves was most efficient at frequencies between 5.16 and $5.45 \mathrm{GHz}$ [18]. BLS was used to image spin waves propagating at the frequency of $5.26 \mathrm{GHz}$ through the multiplexer magnetized by the field of the same value applied at $7^{\circ}$ to the leg length. The resulting snapshots of spin-wave propagation taken at $130 \mathrm{~ns}, 160 \mathrm{~ns}$ and $200 \mathrm{~ns}$ after the onset of the excitation are shown in Figs. 2 (a)-(c), respectively, and clearly show the majority of the spin wave energy flows into the right arm of the multiplexer.

Upon diffraction of the incident spin waves at the leg-arm junction [19], spin waves with a range of $k_{\mathrm{x}}$ values are generated. Since the isofrequency curve is approximately straight for $k_{\mathrm{x}} \geq 5 \mathrm{~mm}^{-1}$ (Fig. 1 (c)), the group velocities of these spin waves are collinear, leading to the generation of spin-wave caustic beams [19],[20]. The tilt of the bias magnetic field also induces asymmetry in the power distribution of spin waves excited by the microstrip antenna in the leg, as shown in the upper panel of Fig. 1 (c), which leads to the observed asymmetry in the relative amplitude of spinwave beams in the left and right arms of the multiplexer. The snake-like propagation path of the spin waves originates from the incident spin wave (with wave vector $\boldsymbol{k}_{\mathrm{i}}$ and group velocity $\boldsymbol{v}_{\mathrm{g}, \mathrm{i}}$ ) reflecting from the edges of the multiplexer arm (giving rise to a spin wave of wave vector $\mathbf{k}_{\mathrm{r}}$ and group velocity $\boldsymbol{v}_{\mathrm{g}, \mathrm{r}}$ ).
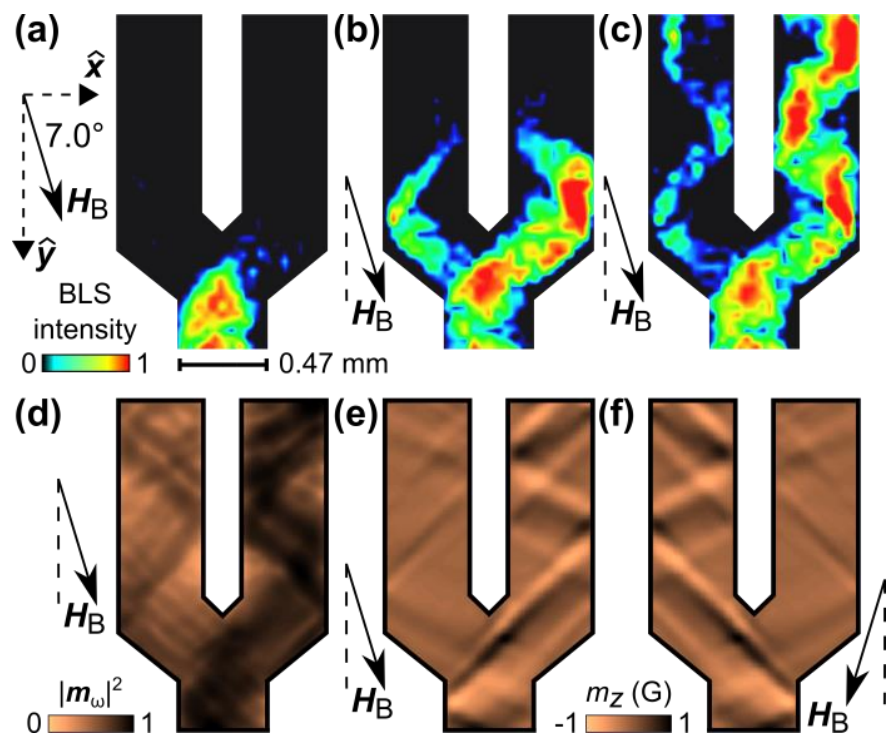

Fig. 2 (color online) (a)-(c) BLS snapshots of amplitude distribution are shown for a $5.26 \mathrm{GHz}$ spin wave propagating down the multiplexer at $120 \mathrm{~ns}, 160 \mathrm{~ns}$ and $200 \mathrm{~ns}$ after the onset of the microwave excitation at the base of the multiplexer leg, respectively. (d) The spin-wave amplitude distribution (comparable to the BLS snapshots) calculated from micromagnetic simulations is shown for the frequency of $5.09 \mathrm{GHz}$. (e)-(f) Snapshots of the dynamic out-of-plane component of magnetization $m_{\mathrm{z}}$ are shown for the bias magnetic field applied at $7^{\circ}$ and $-7^{\circ}$ relative to the multiplexer leg, respectively. The spin waves are continuously excited by a harmonic microwave field of $5.09 \mathrm{GHz}$ frequency.
The experimental results are fully corroborated by the micromagnetic calculations (Fig. 2 (d)). The simulations were performed at the frequency of $5.09 \mathrm{GHz}$ due to small inescapable differences between the measured and modeled samples (e.g. edge roughness, idealized simulation parameters etc.) As shown by the simulated snapshots presented in Fig. 2 (e) and (f), by rotating the bias field from $7^{\circ}$ to $-7^{\circ}$ respectively, the spin wave output can be toggled. The simulations also enabled us to extract information about the phase of the steered spin waves. As can be seen from the snapshots shown in Figs. 2 (e)-(f), the spin-wave phase fronts are almost orthogonal to the propagation path, both at the junction and at the arm. The orientation of the group velocity and phase fronts (i.e. phase velocity) is consistent with those expected from the isofrequency curve shown in Fig. 1 (c).

\section{DISCUSSION}

The multiplexer studied here is controlled by the orientation of the global bias magnetic field. However, it would be undesirable to rotate the sample (or the magnet) in practice. Instead, it is envisioned that the global bias magnetic field could be "rotated" by combining of two in-plane magnetic fields aligned along the $x$ and $y$ directions, respectively. Then, the rotation of the net bias magnetic field could be delivered merely by toggling the polarity of the magnetic field along the $x$ axis. For the system considered here, the net bias field is $1160 \mathrm{Oe}$, and so the components along the $x$ and $y$ directions would have to be 141 and $1151 \mathrm{Oe}$, respectively. The $y$ component of the field $(1151 \mathrm{Oe})$ would remain fixed, while its $x$-component $(141 \mathrm{Oe})$ could be toggled between positive and negative $x$ so as to direct the output spin wave along the right or left multiplexer branch, respectively.

The maintenance of the constant bias magnetic field along the long axis of the multiplexer could require some power to sustain. In principle, this could be overcome by exploiting micromagnetic memory, since at $\boldsymbol{H}_{\mathrm{B}}=0$ the structure should still retain its magnetization due to its magnetic shape anisotropy [16]. Alternatively, locally positioned microscale permanent magnets could be employed [21]. Exploration of the challenges is however beyond the scope of this paper.

The conversion of planar spin waves to caustic beams, the phase of which is rather poorly defined, also raises the question as to whether it is possible to convert the caustics back into planar waves. Here, we show that this could be achieved by directing the caustic beams into a waveguide of reduced width. The angular width of the spin wave caustic propagating in the arm of the multiplexer is of the order of $100 \mu \mathrm{m}$. So, the output waveguide needs to be patterned to approximately match this in width. We performed micromagnetic simulations to verify this basic concept of phase velocity conditioning. Fig. 3 (a) shows a snapshot of the out-of-plane component of the magnetization taken $4 \mathrm{~ns}$ after the spin waves of $5.09 \mathrm{GHz}$ frequency were excited harmonically at the base of the multiplexer leg. This time, the spin wave caustic beams were directed into an output waveguide of $100 \mu \mathrm{m}$ width. Since the waveguide is much 
narrower than before, the spin-wave amplitude is now much more uniform across its width. The phase fronts are observed to be nearly orthogonal (yet slightly angled but much less so as compared to the case of the $500 \mu \mathrm{m}$ wide waveguide) relative to the waveguide length and therefore the direction of propagation.
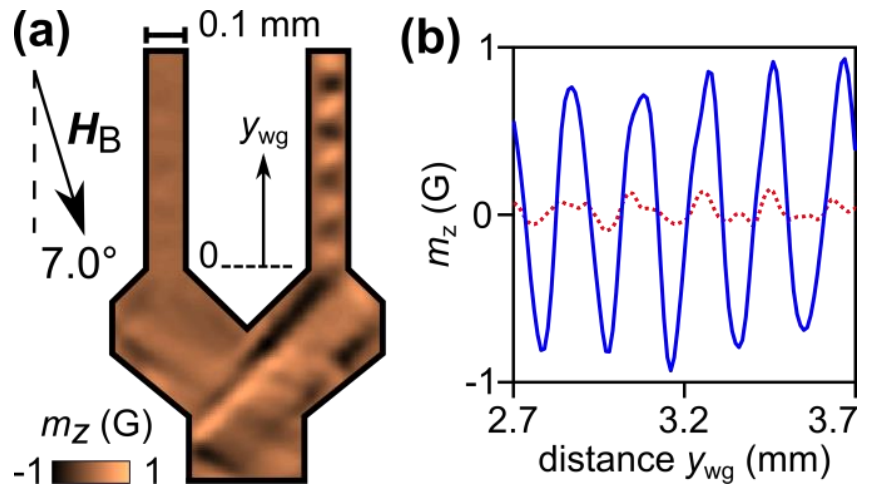

Fig. 3 (a) The out-of-plane component of the dynamic magnetization $m_{\mathrm{z}}$ is shown for a $5.09 \mathrm{GHz}$ spin wave propagating down the multiplexer at about $4 \mathrm{~ns}$ after harmonic field excitation. (b) The simulated spatial variation of $m_{\mathrm{z}}$ is shown for a portion of the left (dashed red) and right (solid blue) output arms of the multiplexer. The bias magnetic field is oriented at $7^{\circ}$ from the vertical symmetry axis of the multiplexer. The distance $\mathrm{y}_{\mathrm{wg}}$ is measured from the armwaveguide junction.

Photonic waveguides benefit substantially from the extremely low dissipation of light in glass. In magnonic waveguides however, the spin wave damping can be much more significant. Yet, it is known that planar spin waves are capable of propagating for tens of millimeters in YIG. To evaluate the path length of the continuously excited spin waves with partly entangled phase, we performed further simulations for a multiplexer with output waveguide arms of extended length: the output waveguides had length of about 8 mm. Fig. 3 (b) shows the dynamic out-of-plane component of magnetization along the left and right waveguides averaged across their widths. The wavelength of the output quasi-plane spin wave is observed to be around $200 \mu \mathrm{m}$. No additional (e.g. due to dephasing) decrease in amplitude of the spin waves was observed over the waveguide length of $8 \mathrm{~mm}$. The ratio of the spin wave amplitudes in the right and left output waveguides was observed to be of the order of $6: 1$.

\section{CONCLUSIONS}

We have used BLS imaging experiments and OOMMF micromagnetic simulations to demonstrate functionality of a Y-shaped YIG magnonic multiplexer control by static uniform bias magnetic field. We have shown that the spin wave beam can be switched between the output arms of the multiplexer by toggling the direction of a magnetic field as small as 141 Oe. Finally, we have demonstrated that the spin-wave phase at the output arm of the multiplexer could be conditioned by reducing the arm width to about the width of the spin wave caustic beam. Our results are relevant to the development of spin-wave based ("magnonic") devices for data and high frequency signal processing.

\section{ACKNOWLEDGMENT}

The research leading to these results has received funding from the Engineering and Physical Sciences Research Council (EPSRC) of the United Kingdom (Project Nos. EP/L019876/1 and EP/P505526/1), the Russian Science Foundation (Project No. 14-19-00760), the scholarship of the President of the Russian Federation (Project No. SP-313.2015.5) and the Russian Foundation for Basic Research (Project No. 14-0700273).

\section{REFERENCES}

[1] International Technology Roadmap for Semiconductors (ITRS) 2013 edition: Emerging Research Devices. http://www.itrs.net/Links/2013ITRS/Summary2013.htm (accessed 7 March 2015).

[2] A. Hirohata and K. Takanashi, J. Phys. D: Appl. Phys. 47, 193001 (2014).

[3] V. V. Kruglyak, S. O. Demokritov, and D. Grundler, J. Phys. D - Appl. Phys. 43, 264001 (2010).

[4] R. L. Stamps et al., J. Phys. D: Appl. Phys. 47, 333001 (2014).

[5] S. V. Vasiliev, V. V. Kruglyak, M. L. Sokolovskii, and A. N. Kuchko, J. Appl. Phys. 101, 113919 (2007).

[6] K.-S. Lee and S.-K. Kim, J. Appl. Phys. 104, 053909 (2008).

[7] A. Khitun, M. Bao, and K. L. Wang, J. Phys. D - Appl. Phys. 43, 264005 (2010).

[8] K. Vogt et al., Nature Commun. 5, 3727 (2014).

[9] R. W. Damon and J. Eschbach, J. Phys. Chem. Solids 19, 308 (1961).

[10] T. Schneider, A. A. Serga, T. Neumann, B. Hillebrands, and M. P. Kostylev, Phys. Rev. B 77, 214411 (2008).

[11] V. E. Demidov, S. Urazhdin, and S. O. Demokritov, Appl. Phys. Lett. 95, 262509 (2009).

[12] Y. Au et al., Appl. Phys. Lett. 98, 122506 (2011).

[13] S. O. Demokritov, B. Hillebrands, and A. N. Slavin, Phys. Rep. 348, 441 (2001).

[14] A. V. Sadovnikov et al., Appl. Phys. Lett. 106, 192406 (2015)

[15] M. Donahue and D. Porter, Interagency Report NISTIR 6376 (NIST, Gaithersburg, MD, 1999).

[16] M. Dvornik, Y. Au and V. V. Kruglyak, Top. Appl. Phys. 125, 101 (2013).

[17] O. Karlqvist, Trans. Roy. Inst. Techno. 86, 3 (1954).

[18] C. S. Davies et al., "Toward graded-index magnonics: steering spin waves across magnonic networks," unpublished.

[19] T. Schneider et al., Phys. Rev. Lett. 104, 197203 (2010).

[20] V. E. Demidov et al., Phys. Rev. B 80, 014429 (2009).

[21] L. O'Brien et al., Phys. Rev. Lett. 106, 087204 (2011). 